This is an electronic reprint of the original article. This reprint may differ from the original in pagination and typographic detail.

Author(s): Silaev, Mikhail; Garaud, Julien; Babaev, Egor

Title: $\quad$ Phase diagram of dirty two-band superconductors and observability of impurityinduced $\mathrm{s}+$ i s state

Year: $\quad 2017$

Version:

Please cite the original version:

Silaev, M., Garaud, J., \& Babaev, E. (2017). Phase diagram of dirty two-band superconductors and observability of impurity-induced s + i s state. Physical Review B, 95(2), 024517. https://doi.org/10.1103/PhysRevB.95.024517

All material supplied via JYX is protected by copyright and other intellectual property rights, and duplication or sale of all or part of any of the repository collections is not permitted, except that material may be duplicated by you for your research use or educational purposes in electronic or print form. You must obtain permission for any other use. Electronic or print copies may not be offered, whether for sale or otherwise to anyone who is not an authorised user. 


\title{
Phase diagram of dirty two-band superconductors and observability of impurity-induced $s+i s$ state
}

\author{
Mihail Silaev, ${ }^{1,2}$ Julien Garaud, ${ }^{1}$ and Egor Babaev ${ }^{1}$ \\ ${ }^{1}$ Department of Theoretical Physics, KTH-Royal Institute of Technology, Stockholm, SE-10691 Sweden \\ ${ }^{2}$ Department of Physics and Nanoscience Center, University of Jyväskylä, Post Office Box 35 (YFL), FI-40014 Jyväskylä, Finland
}

(Received 18 October 2016; revised manuscript received 15 December 2016; published 30 January 2017)

\begin{abstract}
We investigate the phase diagram of dirty two-band superconductors. This paper primarily focuses on the properties and observability of the time-reversal symmetry-breaking $s+i s$ superconducting states, which can be generated in two-band superconductors by interband impurity scattering. We show that such states can appear in two distinct ways. First, according to a previously discussed scenario, the $s+i s$ state can form as an intermediate phase at the impurity-driven crossover between $s_{ \pm}$and $s_{++}$states. We show that there is a second scenario where domains of the $s+i s$ state exists in the form of an isolated dome inside the $s_{ \pm}$domain, completely detached from the transition between $s_{ \pm}$and $s_{++}$states. We demonstrate that in both cases the $s+i s$ state generated by impurity scattering exists in an extremely small interval of impurity concentrations. Although this likely precludes direct experimental observation of the $s+i s$ state formation due to this mechanism, this physics leads to the appearance of a region inside both the $s_{ \pm}$and $s_{++}$domains with unusual properties due to softening of normal modes.
\end{abstract}

DOI: 10.1103/PhysRevB.95.024517

\section{INTRODUCTION}

The physics of unconventional superconductors with multiple broken symmetries has attracted a lot of attention for a long time [1]. Of particular interest is the broken time-reversal symmetry state (BTRSS) which has been proposed to exist in $\mathrm{Sr}_{2} \mathrm{RuO}_{4}$ [2,3] and heavy-fermion compounds [4]. In these systems the discussed BTRSS is commonly referred to as the $p+i p$ state by analogy with the chiral A phase of superfluid ${ }^{3} \mathrm{He}$ confined in a thin slab $[1,5]$. There, the BTRSS appears in combination with parity breaking.

In multiband systems, a different type of time-reversal symmetry-breaking state, termed $s+i s$, can appear. It is fundamentally different from the well-studied $p+i p$ state, and its principal distinction is that it does not break any crystal symmetries and thus represents a type of superconducting state beyond the lattice point group-based classification. Such states were discussed in a wide range of systems, and in particular in three-band superconductors with frustrated interband interactions [6-8].

The $s+i s$ states have been predicted to host a broad range of interesting new phenomena, among which can be mentioned different massless [9] and "phase-density mixed" [8,10-12] collective modes, unconventional thermoelectric properties $[13,14]$, additional mechanisms of damping of the vortex motion [15], and unconventional magnetic signatures induced by defects $[16,17]$. Multiple broken symmetries in $s+i s$ superconductors give rise to several strongly disparate coherence lengths. This can lead to a state with attractive intervortex interaction originating in the magnetic field penetration length being smaller than some, and larger than other coherence lengths [10,18]. Besides vortices, the $s+i s$ state also allows other types of topological excitations that include domain walls and skyrmions [19-21]. The $s+i s$ state also exhibits complex beyond-mean-field physics with new fluctuation-induced phases [22-25].

The $s+i s$ state can have various microscopic physics origins. It was recently shown to be generated by quasiparticle scattering in multiband superconductors with repulsive interaction of electrons in different bands [26,27]. This mechanism would be rather generic for iron-pnictide superconductors, where the pairing is generated by the interband electron-electron repulsion [28], producing the so-called $s_{ \pm}$ superconducting state with a sign change between the orderparameter components in different bands [29,30]. That is, for two components of order parameter $\left|\Delta_{i}\right| e^{i \varphi_{i}}$, the state has $\varphi_{1}=\varphi_{2}+\pi$ in contrast to the $s_{++}$state where $\varphi_{1}=\varphi_{2}$.

Existence of the $s+i s$ state localized near the surface of a two-band $s_{ \pm}$superconductor has been investigated in [26], while it was later proposed that interband impurity scattering can generate the $s+i s$ state in the bulk [27]. It has further been argued that the disorder-induced transition from $s_{ \pm}$to $s_{++}$state in two-band superconductors can occur in two qualitatively different ways. The first one is a crossover without additional symmetry breaking when the superconducting gap in one of the bands crosses zero, as a function of impurity concentration [31]. The second possible scenario involves $s_{ \pm}$ to $s_{++}$state transformation through the intermediate complex $s+i s$ state $\varphi_{1}=\varphi_{2}+\delta$ with $\delta \neq 0, \pi$ [27].

In this paper we calculate phase diagrams of superconducting states in the presence of interband scattering, and discuss the basic properties of the emerging BTRSS. We point out that in general the phase diagrams are quantitatively and also qualitatively different from the one sketched in [27]. In particular we show that a domain of the $s+i s$ state is not necessarily attached to the $s_{ \pm}$to $s_{++}$crossover line but under certain conditions arises inside the $s_{ \pm}$state. We further analyze, within the framework of microscopic GinzburgLandau expansion, the properties of the transition lines to the $s+i s$ state. Finally, we conclude that in the superconductors described by a weak-coupling two-band theory $s+i s$ occupies a very narrow region of the phase diagram as a function of impurity concentration and temperature. Thus, in such two-band systems, it is extremely unlikely to observe the impurity-induced $s+i s$ states. However, its presence on the phase diagram can influence the properties of the superconducting state in a wider region of the phase diagram even outside the $s+i s$ domain, as we discuss below. 
The rest of this paper is organized as follows. Section II introduces the basic framework and briefly discusses the known properties of impurity-driven $s_{ \pm}$to $s_{++}$crossover in two-band superconductors. Next, Sec. III presents the numerically calculated phase diagrams, in several characteristic cases. Section IV addresses the general mean-field properties of the $s+i s$ transition regions. The mean-field critical behavior at the $s_{ \pm} / s+i s$ and $s_{++} / s+i s$ transition lines is discussed in terms of the Ginzburg-Landau theory in Sec. V, and finally our conclusions are given in Sec. VI.

\section{MICROSCOPIC MODEL}

We consider a superconductor with two overlapping bands at the Fermi level. Within the quasiclassical approximation, the band parameters characterizing the two different sheets are the partial densities of states $n_{k}$, labeled by the band index $k=$ 1,2 . The order parameter is determined, using a microscopic theory formulated in terms of quasiclassical propagators $g_{k}$ and $f_{k}$, the normal and anomalous Green's functions obeying the normalization condition $\left|f_{k}\right|^{2}+g_{k}^{2}=1$. The system of Eilenberger equations for a spatially homogeneous two-band superconductor with impurities reads as [32]

$$
\begin{aligned}
& \omega_{n} f_{1}=\Delta_{1} g_{1}+\gamma_{12}\left(g_{1} f_{2}-g_{2} f_{1}\right), \\
& \omega_{n} f_{2}=\Delta_{2} g_{2}+\gamma_{21}\left(g_{2} f_{1}-g_{1} f_{2}\right),
\end{aligned}
$$

where $\omega_{n}=(2 n+1) \pi T, n \in \mathbb{Z}$ are the fermionic Matsubara frequencies, $T$ is the temperature, and $\gamma_{k k^{\prime}}$ are the interband scattering rates proportional to the impurity concentration. The components of the order parameter $\Delta_{k}=\left|\Delta_{k}\right| e^{i \varphi_{k}}$ are determined by the self-consistency equation

$$
\Delta_{k}=2 \pi T \sum_{n=0}^{N_{d}} \sum_{k, k^{\prime}=1,2} \lambda_{k k^{\prime}} f_{k^{\prime}}\left(\omega_{n}\right)
$$

for the Green's function and satisfy Eqs. (1) and (2). Here $N_{d}=$ $\Omega_{d} /(2 \pi T)$ is the summation cutoff at Debye frequency $\Omega_{d}$. The diagonal elements $\lambda_{k k}$ of the coupling matrix $\hat{\Lambda}$ in the selfconsistency equation (3) describe the intraband pairing, while the interband interaction is determined by the off-diagonal terms which can be either positive or negative. In the following, we consider the latter case which corresponds to the interband repulsion, favoring the sign changing $s_{ \pm}$state. The interband coupling parameters and impurity scattering amplitudes satisfy the symmetry relation [32]

$$
\lambda_{i j}=-\lambda_{J} / n_{i} \quad \text { and } \quad \gamma_{i j}=n_{j} \Gamma,
$$

where $\lambda_{J}>0$ and $n_{1,2}$ are the partial densities of states in the two bands.

In general, the $s_{ \pm}$state is not favored by the impurity scattering, which tends to average out the order parameter over the whole Fermi surface, suppressing the critical temperature. Still, provided the interband pairing interaction is weak, superconductivity can be transformed into a $s_{++}$state and survive even in the limit $\Gamma \gg T_{c 0}$, characterized by the critical temperature $T_{c \infty}$ which reads as $[27,28]$

$$
\ln \left(T_{c 0} / T_{c \infty}\right)=n_{1}\left(w_{11}+w_{12}\right)+n_{2}\left(w_{22}+w_{21}\right),
$$

where $T_{c 0}$ is the critical temperature without interband scattering, $\hat{w}=\hat{\Lambda}^{-1}-\lambda^{-1} \hat{I}$, and $\lambda$ is the maximal eigenvalue of the coupling matrix $\hat{\Lambda}$ with the elements $\lambda_{k k^{\prime}}$. According to Eq. (5), one can see that the interband interaction $\lambda_{J}$ should be sufficiently weak, in order to avoid a drastic suppression of the critical temperature in the $s_{++}$state. To derive the criterion note that $n_{1} w_{11}+n_{2} w_{22}>0$, so that the right-hand side of Eq. (5) is larger than $n_{1} w_{12}+n_{2} w_{21}=\lambda_{J} /\left(\lambda_{11} \lambda_{22}\right)$. Therefore in order to have $T_{c \infty}$ not much smaller than $T_{c 0}$, we require the following condition to be fulfilled:

$$
\lambda_{J} /\left(\lambda_{11} \lambda_{22}\right)<1 .
$$

Below we study the phase diagrams, given by the formalism of Eqs. (1)-(3), as functions of $T$ and $\Gamma$, for various pairing coefficients $\lambda_{k k^{\prime}}$. The restriction on pairing interactions [Eq. (6)] will be shown to set up rather strong limitations on the size of the $s+i s$ domains as a function of the effective impurity concentration $\Gamma$.

\section{PHASE DIAGRAMS}

We construct phase diagrams in the plane of parameters $\Gamma, T$ of a two-band superconductor with interband impurity scattering. For that purpose, we solve numerically the system of Eilenberger equations (1) and (2) together with the selfconsistency equation (3), according to the procedure described in the Appendix. The results are shown in Fig. 1, which demonstrates the role of impurities on the state properties, for various representative cases. The different rows in Fig. 1, respectively, display (A) nearly degenerate bands with $\lambda_{11}=$ $0.3, \lambda_{22}=0.29$, (B) intermediate band disparity $\lambda_{11}=0.3$, $\lambda_{22}=0.25$, and (C) strong band disparity $\lambda_{11}=0.3, \lambda_{22}=$ 0.2 . For each of these, the different columns show, respectively, weak (1), intermediate (2), and strong (3) interband pairing interactions (as compared to the intraband couplings).

The crossover line between $s_{ \pm}$and $s_{++}$states, indicated by the thick solid lines in Fig. 1, is associated with the vanishing of $\Delta_{2}$ as we choose $\lambda_{11}>\lambda_{22}$. Everywhere else, both components of the order parameter remain finite. Figure 2 displays the total density $\varrho$, defined as $\varrho^{2}=\left|\Delta_{1}\right|^{2}+\left|\Delta_{2}\right|^{2}$, that corresponds to the various regimes shown in the phase diagrams Fig. 1. The crossover generically occurs for temperatures close to $T_{c}(\Gamma)$ and at lower temperatures the $s_{ \pm}$and $s_{++}$domains in the $(\Gamma, T)$ phase diagram may be separated by an intermediate $s+i s$ state. When this phase is realized, it shows up as a dome that extends down to $T=0$. As discussed below, in Sec. V, both the $s_{ \pm} / s+i s$ and $s_{++} / s+i s$ transition lines are of the second order, at the mean-field level.

The $s+i s$ state is characterized by a relative phase that differs for zero or $\pi$. That possibility can be understood heuristically in the following way: while the interband pairing enforces a $\pi$ phase difference at zero impurities, the impurity scattering favors a phase difference of zero. As the interband pairing and impurity scattering favor different values of the phase locking, the system has to compromise between those behaviors and it can happen that the optimal phase locking is neither zero nor $\pi$ : the $s+i s$ state.

In general the $s+i s$ dome exists at temperature $T<T_{c}$. However, for nearly degenerate bands it starts very close to $T_{c}$ [see Fig. 1(A1)]. As demonstrated in the next section, 
in case of exact degeneracy $\lambda_{11}=\lambda_{22}$, the transition from $s_{ \pm}$to $s_{++}$always occurs through the BTRSS domain, which extends to the $T=T_{c}(\Gamma)$ curve of the $(T, \Gamma)$ diagram. Increasing the disparity of intraband coupling constant $\lambda_{11}-\lambda_{22}$ disconnects the $s+i s$ dome from the $T_{c}(\Gamma)$ curve, replacing it by the crossover line in a certain temperature interval. For small disparity $\lambda_{11}-\lambda_{22} \ll \lambda_{11}$ one can show that the $s+i s$ domain starts at $T^{*} \propto T_{c}\left[1+\alpha\left(\lambda_{11}^{-1}-\lambda_{22}^{-1}\right)\right]$ where $\alpha \sim 1$. This tendency agrees with that shown in the first column of Fig. 1. Simultaneously with the $T^{*}$ suppression, the band disparity extends the crossover line down to lower temperature which finally goes to $T=0$ eliminating the domain of the $s+i s$ state attached to the $s_{ \pm} \rightarrow s_{++}$crossover line.

However, in contrast to the phase diagrams reported in [27], we show that the $s+i s$ state forms under more general conditions as an isolated dome inside the $s_{ \pm}$region on the phase diagram, entirely detached from the $s_{ \pm} \rightarrow s_{++}$crossover line. This effect is demonstrated in the second column of Fig. 1, where we take a larger value of the interband coupling in order to increase the width of the $s+i s$ region, to make it more visible on the diagrams. On the other hand this set of plots demonstrates the general tendency governing the size of the BTRS domain which grows with increased interband coupling. At the same time, however, the critical temperature of the $s_{++}$ state is exponentially suppressed, according to Eq. (5), so that basically the relevant values of $\lambda_{12}$ are restricted by Eq. (6) which does not allow increasing significantly the size of the BTRS domain in the phase diagram.

\section{PROPERTIES OF THE $s+i s$ DOMAIN}

The $s+i s$ state is formed quite generically in case of nearly degenerate bands near the impurity-driven $s_{ \pm} / s_{++}$crossover. However, we find that it occupies only a vanishingly small region of the phase diagrams. In the case of weak interband pairing, defined according to Eq. (6) as $\lambda_{J}<\lambda_{11} \lambda_{22}$ (see first column in Fig. 1), the two lines of second-order phase transitions $s_{ \pm} \rightarrow s+i s$ and $s+i s \rightarrow s_{++}$almost overlap. There is actually a very narrow region in between that requires rather extreme fine tuning of material parameters, as can be seen in a close-up view in Fig. 3.

On the other hand, as can be seen, for example, in the second column of Fig. 1, it appears that increasing the interband interaction tends to widen the region where the $s+i s$ state is realized. However, this growth is limited by the strong $T_{c}$ suppression at the $s_{ \pm} / s_{++}$crossover which sets the upper limit for $\lambda_{J}$ [Eq. (6)]. Hence for all possible values of interband pairing the $s+i s$ domain still represents a vanishingly small region of the phase diagrams. Besides that, the $s+i s$ transition lines go almost parallel to the $T$ axis, and therefore can be detected only by changing the effective impurity concentration, which would make it extremely challenging to realize the $s+i s$ states via this mechanism. Note also that

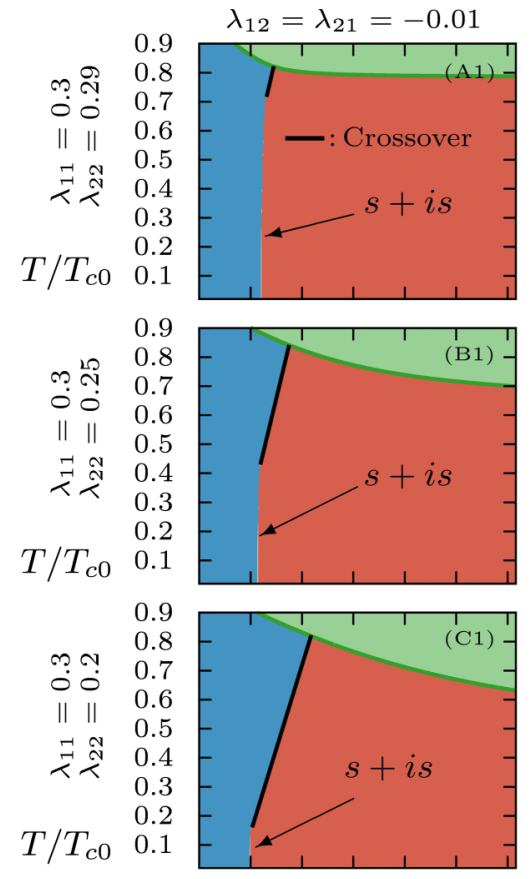

$\begin{array}{rrrrr}0 & 0.2 & 0.4 & 0.6 \\ & & \Gamma\end{array}$
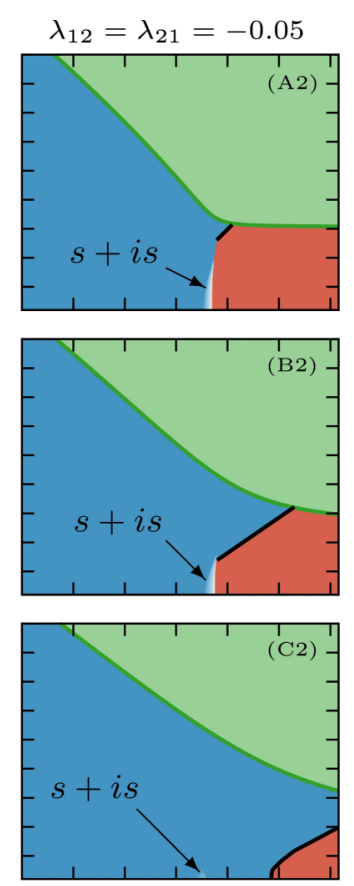

$\Gamma$

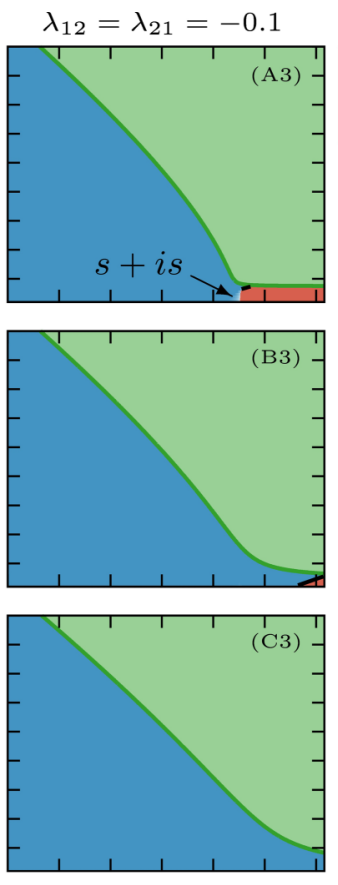

$\Gamma$

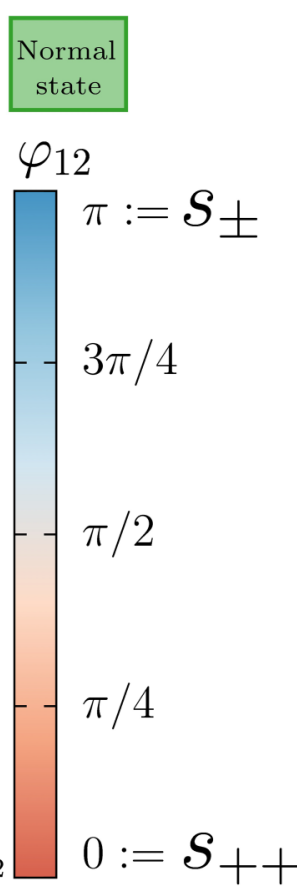

FIG. 1. Phase diagrams of two-band superconductors with interband impurity scattering. These show the values of the lowest-energy-state relative phase $\varphi_{12}=\varphi_{2}-\varphi_{1}$ between the components of the order parameter, as function of temperature and interband scattering $\Gamma$. Different lines show different values of the intraband coupling parameters $\lambda_{11}$ and $\lambda_{22}$, while different columns correspond to different values of the interband couplings $\lambda_{12}$ and $\lambda_{21}$. The green solid line shows the critical temperature of the superconducting phase transition $T_{c}(\Gamma)$ and the solid black line shows the zero of $\Delta_{2}$, that is the crossover between $s_{ \pm}$and $s_{++}$states. In panels A1-A3, B1, B2, and C1, the crossover line is attached to the $s+i s$ dome. In the panel $\mathrm{C} 2$, the crossover line is detached from the $s+i s$ dome, while in the panel $\mathrm{B} 3$ it exists without an $s+i s$ dome. 


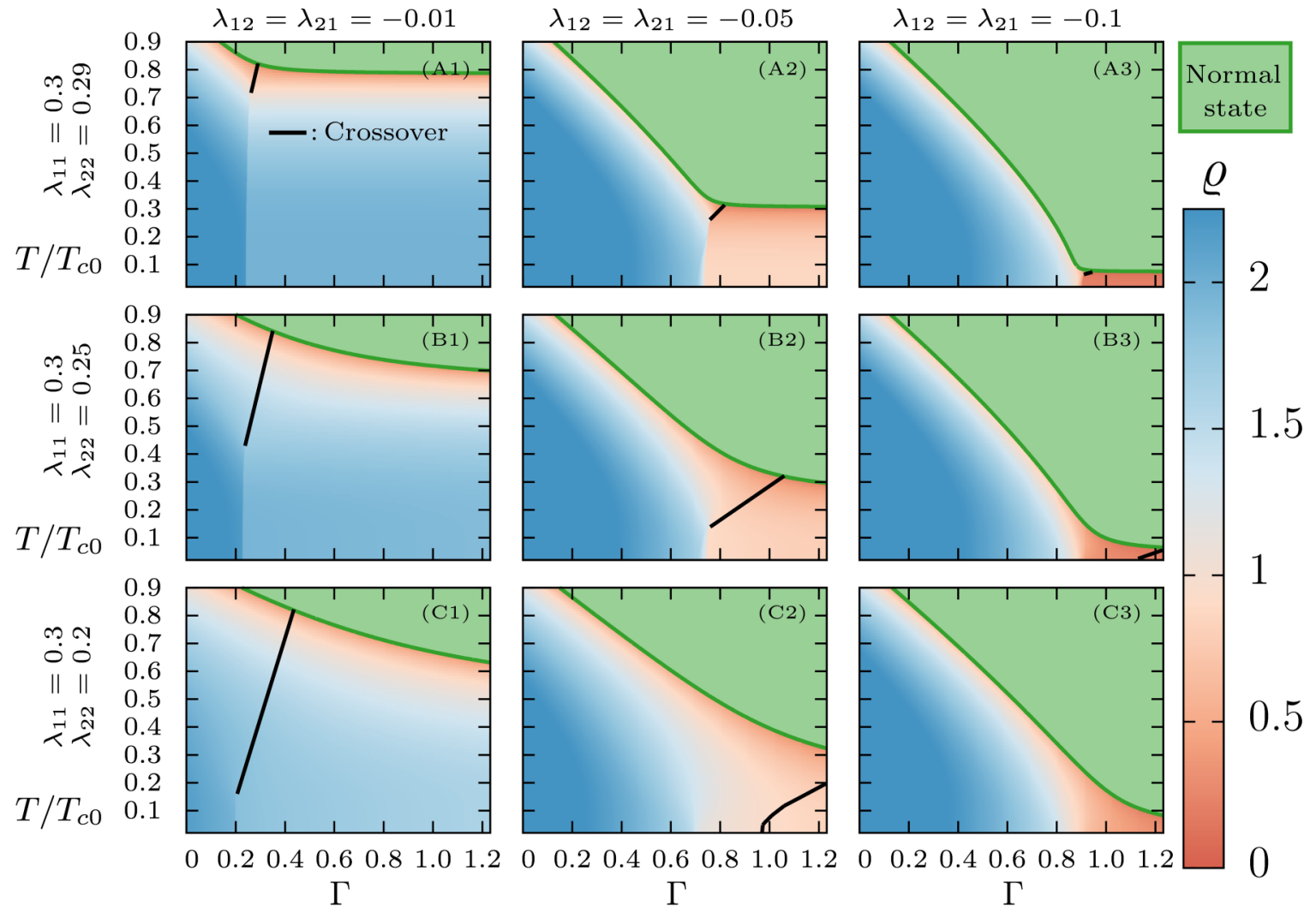

FIG. 2. The total density $\varrho$, defined as $\varrho^{2}=\left|\Delta_{1}\right|^{2}+\left|\Delta_{2}\right|^{2}$ in two-band superconductors with interband impurity scattering, as a function of temperature and interband scattering $\Gamma$. Different lines show different values of the intraband coupling parameters $\lambda_{11}$ and $\lambda_{22}$, while different columns correspond to different values of the interband couplings $\lambda_{12}$ and $\lambda_{21}$. The green solid line shows $T_{c}(\Gamma)$ and the solid black line shows the zero of $\Delta_{2}$, that is the crossover between $s_{ \pm}$and $s_{++}$states.

increasing the band disparity extends the $s_{ \pm} / s_{++}$crossover line and restricts the impurity-induced $s+i s$ state only to very low temperatures. Below, we study the shape and size of the $s+i s$ domain, in the case of degenerate bands. The conclusions obtained are still qualitatively correct in case of moderate band disparities.

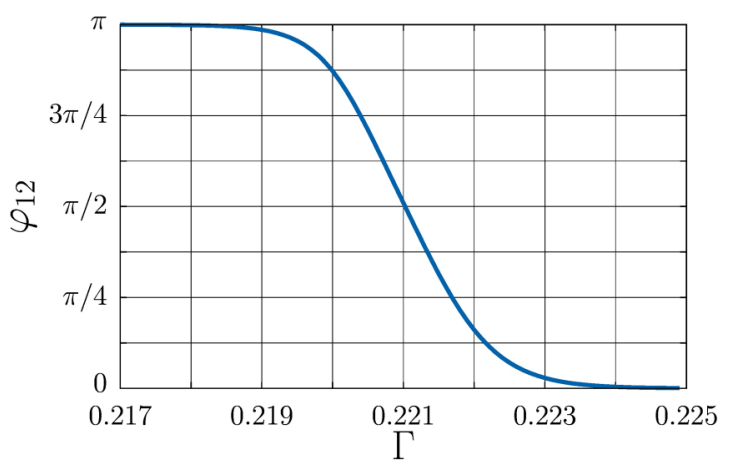

FIG. 3. This shows a close-up view of the transition from $s_{ \pm}$ to $s_{++}$with an intermediate $s+i s$ phase, at $T / T_{c 0}=0.3$ and for parameters corresponding to panel B1 in Fig. 1. This demonstrates that for sufficiently low temperature, in the case of weak interband pairing, the $s+i s$ state is realized as an intermediate state between $s_{ \pm}$and $s_{++}$regions. However, the width of this region is extremely narrow.

\section{A. Size of $s+i$ s transition region: The case of degenerate bands}

To give an analytical estimate of the size of the $s+$ is domain, we consider the simplified case of identical superconducting bands $n_{1}=n_{2}$ so that $\gamma_{12}=\gamma_{21}=\gamma$ and $\lambda_{11}=\lambda_{22}=\lambda$. Under such assumptions, the superconducting gaps can be chosen in a symmetrical form $\Delta_{1}=\Delta_{2}^{*}=\Delta e^{i \varphi}$, so that $f_{1}=f_{2}^{*}=f e^{i \alpha}$, and $g_{1}=g_{2}=g$. Hence the real and imaginary parts of the self-consistency equation (3) become

$$
\begin{array}{r}
2 \pi T \sum_{n=0}^{N_{d}} \frac{g}{\omega_{n}}=\frac{1}{\lambda-\lambda_{J}}, \\
2 \pi T \sum_{n=0}^{N_{d}} \frac{g}{\omega_{n}+2 \gamma g}=\frac{1}{\lambda+\lambda_{J}} .
\end{array}
$$

These equations are simultaneously satisfied in the $s+i s$ phase, while $s_{++}$and $s_{ \pm}$states are described by either Eq. (7) or Eq. (8), respectively.

The transition lines to the $s+i s$ state can be found from the system Eqs. (7) and (8). First of all, we show that in the considered case of exactly degenerate bands, the $s+i s$ domain extends up to the critical temperature. Indeed, at $T=$ $T_{c}$, one can put $g=1$ so that Eqs. (7) and (8) become the definitions of the critical temperature for $s_{++}$and $s_{ \pm}$states, respectively. They are simultaneously satisfied at a single value for interband impurity scattering, $\gamma^{*}$, which determines the 
$s_{ \pm} / s_{++}$transition. Hence according to Eq. (7), the $s+i s$ state exists at this point when $T=T_{c}\left(\gamma^{*}\right)$.

For arbitrary temperatures $T<T_{c}$, the system of Eqs. (7) and (8) can be solved using an expansion by small parameters $\gamma / T_{c 0} \ll 1$ and $\lambda_{J} \ll 1$. The first step is to find the gap amplitudes $\Delta_{+}$and $\Delta_{-}$in $s_{++}$and $s_{ \pm}$states, respectively, with the accuracy up to linear order in $\gamma$ and $\lambda_{J}$. For the $s_{++}$ state we can use the exact expression $g=\omega_{n} / \sqrt{\omega_{n}^{2}+\Delta_{+}^{2}}$. For the $s_{ \pm}$one, we find linear corrections for the propagator using the equation $\left(\omega_{n}+2 \gamma g\right)^{2}\left(1-g^{2}\right)=\Delta_{-}^{2} g^{2}$, which in general does not have an analytical solution. In this way we obtain that the $s_{++}$and $s_{ \pm}$gap amplitudes satisfy

$$
\begin{gathered}
2 \pi T \sum_{n=0}^{N_{d}} \frac{1}{\left(\omega_{n}^{2}+\Delta_{+}^{2}\right)^{1 / 2}}=\frac{1}{\lambda-\lambda_{J}}, \\
2 \pi T \sum_{n=0}^{N_{d}}\left[\frac{1}{\left(\omega_{n}^{2}+\Delta_{-}^{2}\right)^{1 / 2}}-\frac{2 \gamma \omega_{n}^{2}}{\left(\omega_{n}^{2}+\Delta_{-}^{2}\right)^{2}}\right]=\frac{1}{\lambda+\lambda_{J}} .
\end{gathered}
$$

To find the boundaries of the $s+i s$ domain we subtract Eqs. (7) and (8) from each other to obtain

$$
\begin{aligned}
& 2 \pi T \sum_{n=0}^{N_{d}}\left[\frac{\gamma_{+}}{\left(\omega_{n}^{2}+\Delta_{+}^{2}\right)}-\frac{2 \gamma_{+}^{2}}{\left(\omega_{n}^{2}+\Delta_{+}^{2}\right)^{3 / 2}}\right]=\frac{\lambda_{J}}{\lambda^{2}}, \\
& 2 \pi T \sum_{n=0}^{N_{d}}\left[\frac{\gamma_{-}}{\left(\omega_{n}^{2}+\Delta_{-}^{2}\right)}+\frac{2 \gamma_{-}^{2}\left(\Delta_{-}^{2}-\omega_{n}^{2}\right)}{\left(\omega_{n}^{2}+\Delta_{-}^{2}\right)^{5 / 2}}\right]=\frac{\lambda_{J}}{\lambda^{2}},
\end{aligned}
$$

where Eqs. (11) and (12) yield the implicit equations $\gamma_{+}=$ $\gamma_{+}(T)$ and $\gamma_{-}=\gamma_{-}(T)$ describing the $s_{++} / s+i s$ and $s_{ \pm} / s+$ is transition correspondingly. Here we need to take into account the terms up to the second order in $\gamma$ since in the linear order the transition lines coincide.

The largest width for the $s+i s$ domain occurs at low temperatures $T \ll T_{c}$ when we can substitute summation by Matsubara frequencies $2 \pi T \sum_{n}=\int d \omega$. Hence, from Eqs. (9) and (10), we obtain the relation between the gap functions in $s_{++}$and $s_{ \pm}$phases:

$$
\ln \left(\frac{\Delta_{+}}{\Delta_{-}}\right)=\frac{\pi}{2} \frac{\gamma_{c}}{\Delta_{-}}-\frac{2 \lambda_{J}}{\lambda^{2}} .
$$

To the first order in small parameters, Eq. (13) can be reduced to give the difference $\delta \Delta=\Delta_{-}-\Delta_{+}=\gamma \pi / 2$. The relation (13) holds everywhere in the $s_{ \pm}$region, where it gives the dependence of gap function $\Delta_{-}=\Delta_{-}(\gamma)$, while the gap does not depend on $\gamma$ in the $s_{++}$region. Within the same approximation, Eqs. (11) and (12) yield

$$
\begin{aligned}
& \frac{\pi}{2} \frac{\gamma_{+}}{\Delta_{+}}-2 \frac{\gamma_{+}^{2}}{\Delta_{+}^{2}}=\frac{\lambda_{J}}{\lambda^{2}}, \\
& \frac{\pi}{2} \frac{\gamma_{-}}{\Delta_{-}}+\frac{2}{3} \frac{\gamma_{-}^{2}}{\Delta_{-}^{2}}=\frac{\lambda_{J}}{\lambda^{2}} .
\end{aligned}
$$

From the above argument, we conclude that the $s+i s$ domain is located in the vicinity of $\gamma_{c}=2 \Delta_{-} \lambda_{J} /\left(\pi \lambda^{2}\right)$, and the width of the $s+i s$ domain $\delta \gamma=\gamma_{+}-\gamma_{-}$is much smaller, $\delta \gamma \ll \gamma_{c}$. This can be found by combining Eqs. (14) and (15), which yield $\delta \gamma=(0.4 / \pi) \gamma_{c}^{2} / \Delta_{+}$, so that

$$
\delta \gamma=\frac{\Delta_{+}}{20} \frac{\lambda_{J}^{2}}{\lambda^{4}}
$$

For the parameters used in Fig. 3 the estimate (16) yields $\delta \Gamma=2 \delta \gamma \propto 10^{-3}$, which coincides by the order of magnitude with numerically found values. In general Eq. (16) implies that the $s+i s$ domain is generically narrow for this model of a two-band superconductor with impurities since its width is determined by the parameter $\lambda_{J}^{2} / \lambda^{4}$ which is small according to the restriction (6).

The characteristic shape of the $s+i s$ domain in the plots of Fig. 1 can be understood considering one of the transition lines, namely, between $s_{++}$and $s+i s$ domains. With good accuracy we can use the first-order-in- $\gamma$ approximation in Eq. (11), which yields

$$
\frac{\gamma_{+}}{2 \pi T} \psi^{\prime}\left(\frac{\Delta_{+}}{2 \pi T}+\frac{1}{2}\right)=-\frac{\lambda_{J}}{\lambda^{2}},
$$

where $\psi(s)$ is a digamma function satisfying the relation $\psi(s)-\psi(1 / 2)=\sum_{n=0}^{\infty}[1 /(n+1 / 2)-1 /(n+s)]$. Given that the temperature dependence of the gap $\Delta_{+}=$ $\Delta_{+}(T)$ is determined by the usual gap equation (9), one can find that Eq. (17) yields only a weak temperature variation of $\gamma_{+}(T)$ which is consistent with the $s+i s$ domain being elongated, almost parallel to the $T$ axis as can be seen in the first column of Fig. 1.

\section{B. Effect of the band disparity}

We have previously shown that, for exactly degenerate bands, the $s+i s$ state extends all the way up to the critical temperature near the $s_{ \pm}$to $s_{++}$transition. As can be seen from the numerical plots in Fig. 1, for the case of finite band disparity, the $s+i s$ dome is disconnected from the $T_{c}(\Gamma)$ curve. It is instead replaced by a crossover line near the critical temperature. To describe this effect analytically, let us derive the equation describing $s+i$ s transition lines, assuming again that the condition (6) is satisfied, so that the interband scattering amplitudes are small. Here, we are not interested in the width of the $s+i s$ region, so that we implement first-order expansion in $\gamma_{12}$ to obtain from Eqs. (1) and (2)

$$
f_{1}=\Delta_{1} \frac{g_{1}}{\omega_{n}}+\left(\Delta_{2}-\Delta_{1}\right) \frac{\gamma_{12} g_{1} g_{2}}{\omega_{n}^{2}} .
$$

A similar expression for $f_{2}$ is given by the interchange $1 \leftrightarrow 2$ in Eq. (18). Substituting Eq. (18) into the self-consistency equation (3), we find that on both $s_{ \pm} / s+i s$ and $s_{++} / s+i s$ transition lines, the following condition is satisfied:

$$
2 \pi T \sum_{n=0}^{\infty} \frac{g_{1}-g_{2}}{\omega_{n}}=\frac{1}{\lambda_{11}}-\frac{1}{\lambda_{22}},
$$

where we took into account that $\lambda_{12} \lambda_{21} \ll \lambda_{11} \lambda_{22}$.

The relation (19) implies several properties of the $s+i s$ transition lines. First it is clear that the $s+i s$ domain does not reach $T_{c}$, since the condition (19) is not satisfied near the critical temperature where $g_{1}=g_{2}=1$. Besides that, near $T_{c}$ we can put $g_{2}=1$ and $g_{1}=1-\Delta_{1}^{2} / 2 \omega_{n}^{2}$, to rewrite the 
condition (19) in the simpler form

$$
\Delta_{1}^{2}=\frac{8 \pi^{2} T_{c}^{2}}{7 \zeta(3)} \frac{\lambda_{11}-\lambda_{22}}{\lambda_{11} \lambda_{22}},
$$

where $\zeta(3)=1.2$ is the Riemann zeta function. Provided that $\Delta_{1}^{2} \propto T_{c}\left(T_{c}-T\right)$, it is clear that the $s+i s$ states can extend only up to the threshold temperature $T^{*}=T_{c}\left[1+\alpha\left(\lambda_{11}^{-1}-\right.\right.$ $\left.\lambda_{22}^{-1}\right)$ ], where $\alpha \sim 1$. When increasing band disparity, the temperature $T^{*}$ goes down, which agrees with the numerical results displayed in the first column of Fig. 1.

The second important consequence of Eq. (20) follows from the fact that the gap amplitudes $\left|\Delta_{1}\right|$ are different on the $s_{ \pm}$and $s_{++}$sides. Therefore the threshold temperature $T^{*}$ is different for the $s_{ \pm} / s+i s$ and $s_{++} / s+i s$ transition lines. Consequently the phase diagram featuring the $s+i s$ state is in general not only quantitatively but also qualitatively different from the plot given in [27]. Namely, the $s_{ \pm}$to $s_{++}$crossover line is not, in general, attached to the summit of the $s+i s$ dome. Rather, it can attach to an arbitrary point of the line of second order phase transition that separates the $s+i s$ state. This can, for example, be seen for a zoomed in diagram corresponding to panel A3 of Fig. 1.

\section{Domain of $s+i s$ state inside the $s_{ \pm}$phase}

As previously emphasized, in general, the crossover line does not attach to the top of the $s+i s$ dome. We find that it rather attaches to a different point belonging to the $s+i s / s_{++}$ transition line. This implies that the $s+i s$ state also can occur away from the $s_{ \pm}$to $s_{++}$crossover. More precisely, as can be seen in Fig. 4 (corresponding to a zoom on the panel C2 of Fig. 1), the $s+i s$ state can show up as a small "isolated dome" inside the $s_{ \pm}$region. Such an isolated dome can occur for rather important band disparity and intermediate values of the interband impurity scattering. This effect can be understood as follows. When there is band disparity and impurities, the crossover line attaches below the summit of the $s+i s$ dome. Moreover, the $s+i s$ region is generically pushed to lower temperatures when increasing band disparity. It can thus occur that at a certain level of disparity the temperature where the crossover line attaches to the dome goes to zero and then the crossover line detaches from the dome, which means that the $s+i s$ dome becomes isolated.

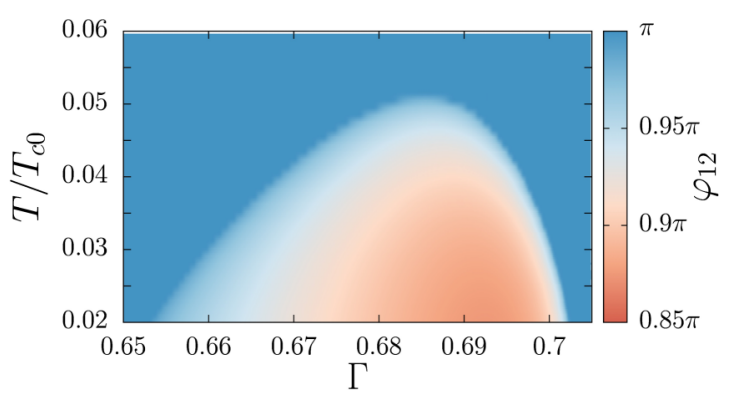

FIG. 4. This displays a close-up view of the "isolated dome" in panel $\mathrm{C} 2$ of Fig. 1. This demonstrates that the $s+i s$ state can actually be disconnected from transition between $s_{ \pm}$and $s_{++}$states.

\section{ORDER OF THE PHASE TRANSITIONS IN THE GINZBURG-LANDAU MODEL}

As discussed in the first part of the paper, the $s+i s$ state, although realized, can be extremely challenging to observe due to the narrowness of the interval in parameter space where this state can exist. Nonetheless, it is a relevant question to study the order of the phase transition to the $s+i s$ state, that is, if the phase transition is of the second order, which guarantees that there appears a divergent coherence length that, in some range of parameters, should exceed the magnetic field penetration length as well as other coherence lengths [10,18]. Regimes with some coherence lengths larger and some smaller than the magnetic field penetration length feature attractive intervortex forces that, under some circumstances, may be responsible for formation of vortex clusters (that kind of regime was termed type-1.5 superconductivity in [33]). Therefore due to the divergent behavior of one of the coherence lengths at the phase transition there may be a range of this state with anomalous magnetic and transport properties.

To determine the order of the phase transition at the meanfield level we derive a Ginzburg-Landau free-energy functional from the microscopic equations. Here we implement the standard multiband expansion in two small gaps $\Delta_{k}=\left|\Delta_{k}\right| \mathrm{e}^{i \varphi_{k}}$ in the dirty case (note that the multiband expansions in general are based on assumptions of several small parameters [34] that are not related to broken symmetries, not to be confused with the simplest expansion in single small parameter $\tau=$ $\left.1-T / T_{c}\right)$. Justification and validity conditions of multiband expansions of this kind in several small parameters were discussed in detail in the clean $s$-wave case [35]. The potential terms in such an expansion read as

$$
\begin{aligned}
\mathcal{F}= & \sum_{k=1}^{2}\left\{a_{k k}\left|\Delta_{k}\right|^{2}+\frac{b_{k k}}{2}\left|\Delta_{k}\right|^{4}\right\} \\
& +2\left(a_{12}+c_{11}\left|\Delta_{1}\right|^{2}+c_{22}\left|\Delta_{2}\right|^{2}\right)\left|\Delta_{1}\right|\left|\Delta_{2}\right| \cos \varphi_{12} \\
& +\left(b_{12}+c_{12} \cos 2 \varphi_{12}\right)\left|\Delta_{1}\right|^{2}\left|\Delta_{2}\right|^{2} .
\end{aligned}
$$

There, the coefficients $a_{k k^{\prime}}, b_{k k^{\prime}}$, and $c_{k k^{\prime}}$ can be calculated from the inputs $\lambda_{k k^{\prime}}, T$, and $\Gamma$ of the microscopic self-consistent equations [27].

We investigate the state properties of the Ginzburg-Landau theory by minimizing the free energy (21) with respect to the densities $\left|\Delta_{k}\right|$ and the relative phase $\varphi_{12}=\varphi_{2}-\varphi_{1}$. The relative phase is given by the equation $\delta \mathcal{F} / \delta \varphi_{12}=0$ :

$$
\begin{aligned}
& \left(a_{12}+c_{11}\left|\Delta_{1}\right|^{2}+c_{22}\left|\Delta_{2}\right|^{2}\right)\left|\Delta_{1}\right|\left|\Delta_{2}\right| \sin \varphi_{12} \\
& \quad+c_{12}\left|\Delta_{1}\right|^{2}\left|\Delta_{2}\right|^{2} \sin 2 \varphi_{12}=0 .
\end{aligned}
$$

This has different solutions in the different states:

$$
\begin{aligned}
s_{ \pm}: \varphi_{12} & =\pi, \quad s_{++}: \varphi_{12}=0 \\
s+i s: \varphi_{12} & = \pm \arccos \left(-\frac{a_{12}+c_{11}\left|\Delta_{1}\right|^{2}+c_{22}\left|\Delta_{2}\right|^{2}}{2 c_{12}\left|\Delta_{1}\right|\left|\Delta_{2}\right|}\right) .
\end{aligned}
$$

Note here that the values of the gaps are determined by the other equations $\frac{\delta \mathcal{F}}{\delta\left|\Delta_{k}\right|}=0$. Figure 5 shows an example of such a calculation applied to the regime displayed in panel A3 of Fig. 1. In the area where the two-band Ginzburg-Landau 


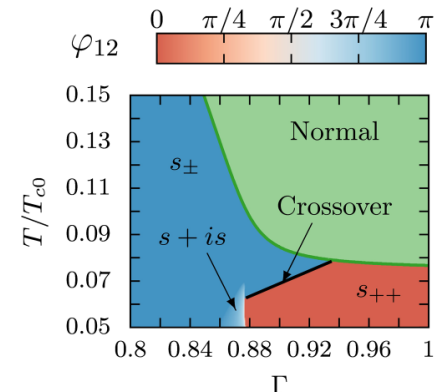

$\Gamma$

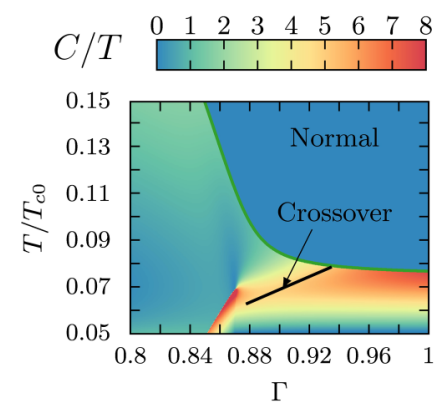

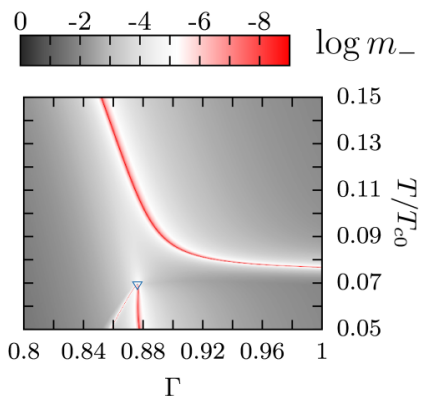

$\Gamma$

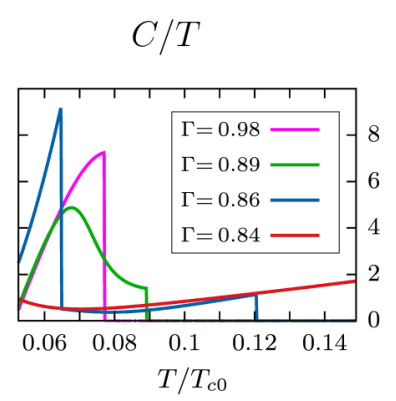

FIG. 5. Top-left panel shows the phase diagram of two-band superconductors with interband impurity scattering for parameters corresponding to the panel A3 in Fig. 1. That is, the values of intraband coupling parameters are $\lambda_{11}=0.3, \lambda_{22}=0.29$, and $\lambda_{12}=$ $\lambda_{21}=-0.1$. The top-right panel displays the smallest eigenvalue of the Hessian matrix of the Ginzburg-Landau free energy. Clearly, it vanishes at $T_{c}$, signaling that the system has a divergent coherence length at these temperatures and thus the second-order phase transition between superconducting and normal state. Moreover, the $s+i s$ phase is surrounded by another line with vanishing smallest eigenvalue, signaling the flatness of the potential and thus an additional second-order transition from $s_{ \pm} / s_{++}$to $s+i s$ states. Note that for the $s_{ \pm} / s_{++}$crossover, all eigenvalues remain finite and the second gap vanishes. The triangle shows the summit of the $s+i s$ dome, where a second eigenvalue vanishes as well. The bottom-left panel shows the corresponding specific heat, while the bottom-right panel displays few one-dimensional cross sections of that plot, corresponding to vertical scans (at a given impurity). There are clearly two jumps when the vertical lines intersect both the superconducting phase transition as well as the phase transition from $s_{ \pm}$to $s+i s$ state.

expansion in small gaps is formally justified, the phase diagram matches with that obtained within the microscopic theory of Eqs. (1)-(3).

Now we focus on the properties of the transition lines between the various phases. For a given state to be stable, all the eigenvalues of the stability (Hessian) matrix $\hat{\mathcal{H}}$ must be positive. The Hessian matrix reads as

$$
\hat{\mathcal{H}}=\left(\begin{array}{ccc}
\frac{\partial^{2} \mathcal{F}}{\partial^{2} \Delta_{1}} & \frac{\partial^{2} \mathcal{F}}{\partial \Delta_{1} \partial \Delta_{2}} & \frac{\partial^{2} \mathcal{F}}{\partial \Delta_{1} \partial \varphi_{12}} \\
\frac{\partial^{2} \mathcal{F}}{\partial \Delta_{1} \partial \Delta_{2}} & \frac{\partial^{2} \mathcal{F}}{\partial^{2} \Delta_{2}} & \frac{\partial^{2} \mathcal{F}}{\partial \Delta_{2} \partial \varphi_{12}} \\
\frac{\partial^{2} \mathcal{F}}{\partial \Delta_{1} \partial \varphi_{12}} & \frac{\partial^{2} \mathcal{F}}{\partial \Delta_{2} \partial \varphi_{12}} & \frac{\partial^{2} \mathcal{F}}{\partial^{2} \varphi_{12}}
\end{array}\right)
$$

and $\mathcal{H}_{a b}$, where $a, b=1,2, \varphi$ denotes the entry of the Hessian that relates to the variations with respect to $\left|\Delta_{1}\right|,\left|\Delta_{2}\right|$, and $\varphi_{12}$, respectively. Second-order phase transitions are associated with a divergent coherence length, and, correspondingly, there should be flatness of the potential in some direction of parameter space. Mathematically, this can be characterized by the vanishing of the smallest eigenvalue of the corresponding Hessian matrix.

By definition, the $s+i s$ state is that where both $\left|\Delta_{1}\right|$ and $\left|\Delta_{2}\right|$ are nonzero and for which $\varphi_{12} \neq 0, \pi$ is given by the equation $\delta \mathcal{F} / \delta \varphi_{12}=0$. Both in the $s_{ \pm}$and $s_{++}$state $\mathcal{H}_{i \varphi}=0$ $(i=1,2)$. And thus the contribution of the relative phases to the stability (Hessian) matrix originates only in $\mathcal{H}_{\varphi \varphi}$. The stability of the $s_{ \pm}$and $s_{++}$states thus requires that $\left.\mathcal{H}_{\varphi \varphi}\right|_{s_{+}, s_{++}}>$ 0 . The transition line to the $s+i s$ is thus given by the condition that $\left.\mathcal{H}_{\varphi \varphi}\right|_{s_{ \pm}, s_{++}}=0$. Indeed, this is the point where $s_{ \pm}$and $s_{++}$ states become unstable and $s+i s$ becomes stable. This gives an additional condition, for the transition line:

$$
\begin{aligned}
& \left(a_{12}+c_{11}\left|\Delta_{1}\right|^{2}+c_{22}\left|\Delta_{2}\right|^{2}\right)\left|\Delta_{1}\right|\left|\Delta_{2}\right| \cos \varphi_{12} \\
& \quad+2 c_{12}\left|\Delta_{1}\right|^{2}\left|\Delta_{2}\right|^{2} \cos 2 \varphi_{12}=0 .
\end{aligned}
$$

The right panel in Fig. 5 displays the smallest eigenvalue of the Hessian matrix of the Ginzburg-Landau free energy. Clearly, it vanishes at $T_{c}$, which means that there is a divergent coherence length and thus the standard, at mean-field level, second-order phase transition between superconducting and normal state. Moreover, the $s+i s$ phase is surrounded by another line with vanishing smallest eigenvalue signaling the flatness of potential, and thus a second-order transition and a divergent coherence length at the transition to the $s+i s$ state. The corresponding behavior of the specific heat $C=$ $-T d S / d T$ following from Eq. (21) is displayed in the bottom line of Fig. 5. By contrast the total density does not have a strong feature at the phase transition (see Fig. 2). Therefore no dramatic variation of magnetic field penetration length is expected.

\section{CONCLUSION}

We discussed the phase diagram of dirty two-band superconductors. Our main interest was to examine the possible occurrence of the $s+i s$ superconducting state in the mean-field model. We find that the state exists and appears under more general circumstances than previously discussed: namely, it is not necessarily connected to the crossover from the $s_{ \pm}$ to $s_{++}$state but can arise inside the $s_{ \pm}$phase. However, we demonstrate that the domain of the $s+i s$ state is extremely small in this model and on a large-scale plot almost shrinks to a line. For all practical purposes that makes it unobservable in experiments in materials with this microscopic physics. We also establish that the phase transitions to the $s+i s$ state are second order on the mean field level. This implies that near the phase transition there is a divergent length scale associated with the order-parameter variations, as well as a related softening of dynamical modes: such as Leggett's [9] and "phase-density mixed" collective modes [8,10-12]. We emphasise that our "nonobservability" results here apply only for the weak-coupling models of two-band superconductivity but do not preclude formation of larger areas of $s+i s$ states by other mechanisms such as three-band systems with frustrated intercomponent interaction. 


\section{ACKNOWLEDGMENTS}

This work was supported by the Swedish Research Council Grant No. 642-2013-7837 and by the Goran Gustafsson Foundation. M.S. was supported in part by the Academy of Finland. The computations were performed on resources provided by the Swedish National Infrastructure for Computing at National Supercomputer Center at Linköping, Sweden.

\section{APPENDIX: NUMERICAL PROCEDURE}

Given a set of microscopic parameters (temperature $T$, interband scattering amplitude $\Gamma$, and couplings $\lambda_{k k^{\prime}}$ ), the state is found by solving numerically the Eilenberger equations (1) and (2), under the condition that $\left|f_{k}\right|^{2}+g_{k}^{2}=1$, together with the self-consistency equation (3), following the procedure described below.

First, note that using Eqs. (1) and (2) the anomalous Green's functions can be expressed in terms of the normal Green's functions, gaps, etc., as

$$
\left(\begin{array}{l}
f_{1} \\
f_{2}
\end{array}\right)=\frac{1}{w}\left(\begin{array}{cc}
g_{1}\left(\omega_{n}+\gamma_{21} g_{1}\right) & \gamma_{12} g_{1} g_{2} \\
\gamma_{21} g_{1} g_{2} & g_{2}\left(\omega_{n}+\gamma_{12} g_{2}\right)
\end{array}\right)\left(\begin{array}{c}
\Delta_{1} \\
\Delta_{2}
\end{array}\right)
$$

with $\quad w=\omega_{n}\left(\omega_{n}+\gamma_{12} g_{2}+\gamma_{21} g_{1}\right)$.

This allows one to find expressions for $\left|f_{k}\right|^{2}$, which once substituted into the normalization condition yields the system of nonlinear equations for the normal Green's functions $g_{k}$, as

$$
\begin{gathered}
w^{2}\left(g_{1}^{2}-1\right)+g_{1}^{2}\left[\left(\omega_{n}+\gamma_{21} g_{1}\right) \operatorname{Re}\left(\Delta_{1}\right)+\gamma_{12} g_{2} \operatorname{Re}\left(\Delta_{2}\right)\right]^{2} \\
\left.+g_{1}^{2}\left[\left(\omega_{n}+\gamma_{21} g_{1}\right) \operatorname{Im}\left(\Delta_{1}\right)+\gamma_{12} g_{2} \operatorname{Im}\left(\Delta_{2}\right)\right]^{2}=0, \quad \text { (A2 }\right) \\
w^{2}\left(g_{2}^{2}-1\right)+g_{2}^{2}\left[\left(\omega_{n}+\gamma_{12} g_{2}\right) \operatorname{Re}\left(\Delta_{2}\right)+\gamma_{21} g_{1} \operatorname{Re}\left(\Delta_{1}\right)\right]^{2} \\
+g_{2}^{2}\left[\left(\omega_{n}+\gamma_{12} g_{2}\right) \operatorname{Im}\left(\Delta_{2}\right)+\gamma_{21} g_{1} \operatorname{Im}\left(\Delta_{1}\right)\right]^{2}=0 .
\end{gathered}
$$

We choose an optimization method to find $g_{1,2}$ from Eqs. (A2) and (A3) based on an objective function $F$ given by

$$
F\left(g_{1}, g_{2}\right)=[\text { Eq. (A2) }]^{2}+[\text { Eq. (A3) }]^{2} .
$$

We use a covariance matrix adaptation evolution strategy [36], which is a stochastic numerical optimization method for nonlinear or nonconvex problems. As compared to other algorithms, this can be rather suboptimal, but since it is a stochastic, gradient-free method, the solution is guaranteed to be independent of any initial guess.

The anomalous Green's functions can be reconstructed, given the solutions $g_{k}\left(\omega_{n}\right)$ of Eqs. (A2) and (A3), using Eq. (A1). Finally, the gaps are constructed using the selfconsistency equation (3). This procedure is iterated via a fixed-point method until the gaps converge according to the criterion that $\sqrt{\sum_{k}\left|\Delta_{k}^{\text {new }}-\Delta_{k}^{\text {old }}\right|^{2}}<10^{-7}$.
[1] Manfred Sigrist and Kazuo Ueda, Phenomenological theory of unconventional superconductivity, Rev. Mod. Phys. 63, 239 (1991).

[2] Andrew Peter Mackenzie and Yoshiteru Maeno, The superconductivity of $\mathrm{Sr}_{2} \mathrm{RuO}_{4}$ and the physics of spin-triplet pairing, $\mathrm{Rev}$. Mod. Phys. 75, 657 (2003).

[3] Wen Huang, Thomas Scaffidi, Manfred Sigrist, and Catherine Kallin, Leggett modes and multiband superconductivity in $\mathrm{Sr}_{2} \mathrm{RuO}_{4}$, Phys. Rev. B 94, 064508 (2016).

[4] Robert Joynt and Louis Taillefer, The superconducting phases of $\mathrm{UPt}_{3}$, Rev. Mod. Phys. 74, 235 (2002).

[5] G.E. Volovik, Quantized Hall effect in superfluid helium-3 film, Phys. Lett. A 128, 277 (1988).

[6] T. K. Ng and N. Nagaosa, Broken time-reversal symmetry in Josephson junction involving two-band superconductors, Europhys. Lett. 87, 17003 (2009).

[7] Valentin Stanev and Zlatko Tešanović, Three-band superconductivity and the order parameter that breaks time-reversal symmetry, Phys. Rev. B 81, 134522 (2010).

[8] Saurabh Maiti and Andrey V. Chubukov, $s+i s$ state with broken time-reversal symmetry in Fe-based superconductors, Phys. Rev. B 87, 144511 (2013).

[9] Shi-Zeng Lin and Xiao Hu, Massless Leggett Mode in ThreeBand Superconductors with Time-Reversal-Symmetry Breaking, Phys. Rev. Lett. 108, 177005 (2012).

[10] Johan Carlström, Julien Garaud, and Egor Babaev, Length scales, collective modes, and type- 1.5 regimes in three-band superconductors, Phys. Rev. B 84, 134518 (2011).

[11] Valentin Stanev, Model of collective modes in three-band superconductors with repulsive interband interactions, Phys. Rev. B 85, 174520 (2012).
[12] M. Marciani, L. Fanfarillo, C. Castellani, and L. Benfatto, Leggett modes in iron-based superconductors as a probe of time-reversal symmetry breaking, Phys. Rev. B 88, 214508 (2013).

[13] Mihail Silaev, Julien Garaud, and Egor Babaev, Unconventional thermoelectric effect in superconductors that break time-reversal symmetry, Phys. Rev. B 92, 174510 (2015).

[14] Julien Garaud, Mihail Silaev, and Egor Babaev, Thermoelectric Signatures of Time-Reversal Symmetry Breaking States in Multiband Superconductors, Phys. Rev. Lett. 116, 097002 (2016).

[15] Mihail Silaev and Egor Babaev, Unusual mechanism of vortex viscosity generated by mixed normal modes in superconductors with broken time reversal symmetry, Phys. Rev. B 88, 220504 (2013).

[16] Saurabh Maiti, Manfred Sigrist, and Andrey Chubukov, Spontaneous currents in a superconductor with $s+i$ s symmetry, Phys. Rev. B 91, 161102 (2015).

[17] Shi-Zeng Lin, Saurabh Maiti, and Andrey Chubukov, Distinguishing between $s+i d$ and $s+i s$ pairing symmetries in multiband superconductors through spontaneous magnetization pattern induced by a defect, Phys. Rev. B 94, 064519 (2016).

[18] J. Garaud, M. Silaev, and E. Babaev, Microscopically derived multi-component Ginzburg-Landau theories for $s+i s$ superconducting state, Physica C: Superconductivity and its Applications, 2016.

[19] Julien Garaud, Johan Carlström, and Egor Babaev, Topological Solitons in Three-Band Superconductors with Broken Time Reversal Symmetry, Phys. Rev. Lett. 107, 197001 (2011). 
[20] Julien Garaud, Johan Carlström, Egor Babaev, and Martin Speight, Chiral $\mathbb{C} P^{2}$ skyrmions in three-band superconductors, Phys. Rev. B 87, 014507 (2013).

[21] Julien Garaud and Egor Babaev, Domain Walls and Their Experimental Signatures in $s+i s$ Superconductors, Phys. Rev. Lett. 112, 017003 (2014).

[22] Troels Arnfred Bojesen, Egor Babaev, and Asle Sudbø, Time reversal symmetry breakdown in normal and superconducting states in frustrated three-band systems, Phys. Rev. B 88, 220511 (2013).

[23] Troels Arnfred Bojesen, Egor Babaev, and Asle Sudbø, Phase transitions and anomalous normal state in superconductors with broken time-reversal symmetry, Phys. Rev. B 89, 104509 (2014).

[24] Johan Carlström and Egor Babaev, Spontaneous breakdown of time-reversal symmetry induced by thermal fluctuations, Phys. Rev. B 91, 140504 (2015).

[25] Alberto Hinojosa, Rafael M. Fernandes, and Andrey V. Chubukov, Time-Reversal Symmetry Breaking Superconductivity in the Coexistence Phase with Magnetism in Fe Pnictides, Phys. Rev. Lett. 113, 167001 (2014).

[26] A. M. Bobkov and I. V. Bobkova, Time-reversal symmetry breaking state near the surface of an $s_{ \pm}$superconductor, Phys. Rev. B 84, 134527 (2011).

[27] Valentin Stanev and Alexei E. Koshelev, Complex state induced by impurities in multiband superconductors, Phys. Rev. B 89, 100505 (2014).

[28] P. J. Hirschfeld, M. M. Korshunov, and I. I. Mazin, Gap symmetry and structure of Fe-based superconductors, Rep. Prog. Phys. 74, 124508 (2011).
[29] I. I. Mazin, D. J. Singh, M. D. Johannes, and M. H. Du, Unconventional Superconductivity with a Sign Reversal in the Order Parameter of $\mathrm{LaFeAsO}_{1-x} \mathrm{~F}_{x}$, Phys. Rev. Lett. 101, 057003 (2008).

[30] A. V. Chubukov, D. V. Efremov, and I. Eremin, Magnetism, superconductivity, and pairing symmetry in iron-based superconductors, Phys. Rev. B 78, 134512 (2008).

[31] D. V. Efremov, M. M. Korshunov, O. V. Dolgov, A. A. Golubov, and P. J. Hirschfeld, Disorder-induced transition between $s_{ \pm}$ and $s_{++}$states in two-band superconductors, Phys. Rev. B 84, 180512 (2011).

[32] A. Gurevich, Enhancement of the upper critical field by nonmagnetic impurities in dirty two-gap superconductors, Phys. Rev. B 67, 184515 (2003).

[33] Victor Moshchalkov, Mariela Menghini, T. Nishio, Q. H. Chen, A. V. Silhanek, V. H. Dao, L. F. Chibotaru, N. D. Zhigadlo, and J. Karpinski, Type-1.5 Superconductivity, Phys. Rev. Lett. 102, 117001 (2009).

[34] D. R. Tilley, The Ginzburg-Landau equations for pure two band superconductors, Proc. Phys. Soc. 84, 573 (1964).

[35] Mihail Silaev and Egor Babaev, Microscopic derivation of two-component Ginzburg-Landau model and conditions of its applicability in two-band systems, Phys. Rev. B 85, 134514 (2012).

[36] Nikolaus Hansen, The CMA evolution strategy: A comparing review, in Towards a New Evolutionary Computation: Advances in the Estimation of Distribution Algorithms, edited by Jose A. Lozano, Pedro Larrañaga, Iñaki Inza, and Endika Bengoetxea (Springer, Berlin, 2006), Chap. 4, p. 75. 